\section{Associação entre prática de atividade física e indicadores de comportamento sexual de risco em adolescentes}

\author{
Association between physical activity \\ and indicators of risk sexual behavior in \\ adolescents
}

\author{
Josias da Costa Pimentel ${ }^{1}$ \\ Carla Menêses Hardman ${ }^{2}$ \\ Rildo de Souza Wanderley Júnior ${ }^{3}$ \\ Simone José dos Santos ${ }^{4}$ \\ Anísio Luiz da Silva Brito ${ }^{4}$ \\ Rodrigo Antunes Lima ${ }^{3}$ \\ Mauro Virgílio Gomes de Barros ${ }^{2,3,4}$
}

\section{Resumo}

O objetivo deste estudo foi analisar a associação entre prática de atividades físicas no lazer (PAFL) e participação nas aulas de Educação Física (PAEF) com indicadores de comportamento sexual de risco (CSR) em estudantes adolescentes. Realizou-se um estudo transversal baseado na análise secundária de dados de uma amostra representativa de estudantes do ensino médio da rede pública estadual de Pernambuco. Dados de 4.207 estudantes (14-19 anos) foram coletados mediante aplicação de um questionário previamente validado (GSHS-WHO). Os adolescentes que relataram não ter usado preservativo na última relação sexual ou ter três ou mais parceiros ao longo da vida foram considerados expostos aos CSR. Nas análises de regressão logística os indicadores de CSR foram definidos como desfechos enquanto a PAFL e PAEF foram consideradas variáveis independentes. Sexo, faixa etária, afiliação religiosa, morar com os pais, consumo de álcool e tabagismo foram fatores incluídos nas análises como covariáveis. Observou-se que os adolescentes que relataram participar de pelo menos uma aula de Educação Física tinham chance $25 \%$ inferior de intercurso sexual sem uso de preservativo quando comparados àqueles que não participavam das aulas. Aqueles que referiram praticar AFL tinham chance $67 \%$ superior de relatarem experiência de intercurso sexual com três ou mais parceiros. A PAEF e a PAFL foram identificados como fatores associados a indicadores de CSR, mas o inesperado sentido da ligação entre PAFL e números de parceiros na vida exige aprofundamento da investigação nesta área.

\section{Palavras-chave}

Comportamento Sexual; Atividade motora; Educação física e treinamento; Adolescentes e estudantes.

\begin{abstract}
The aim of this study was to analyze the association between physical activity during leisure time (PAL) and participation in physical education classes (PEC) with indicators of sexual risk behavior (SRB) in adolescents. It was a cross-sectional study using secondary analysis of data from a representative sample of high school students attended at the state public education system in Pernambuco, Brazil. Data from 4,207 students (14-19 years) were collected by applying a previously validated questionnaire (GSHSWHO). Students who reported not have used a condom in the last intercourse and had three or more partners during their life were considered exposed to indicators of the SRB. Logistic regression was performed with SRB set as dependent variable, PAL and PEC defined as independent variables, and sex, age group, religious affiliation, living with parents, alcohol consumption and smoking as potential confounders. It was observed that adolescents who reported have participated of PEC at least one time per week were $25 \%$ less likely to refer have not used condom in the last sexual intercourse in comparison with those who never participated in physical education classes. In relation to sexual intercourse, those who reported practice of PAL have a $67 \%$ higher likelihood to have had three or more partners in their lifetime sexual experience. It was concluded that PEC and PAL were associated with sexual risk behaviors, but the surprising direct association between PAL practice and number of sexual partners needs to be further investigated to elucidate causal pathways.
\end{abstract}

\section{Keywords}

Sexual behavior; Motor activity; Physical education and training; Adolescents; Students.
Rev Bras Ativ Fis Saúde p. 493-500 DOI:

http://dx.doi.org/10.12820/rbafs.v.18n4p493

1 Universidade Federal de Pernambuco. Residência Multiprofissional em Saúde da Família - UFPE/CAV. Vitória de Santo Antão - PE, Brasil.

2 Universidade Federal de Santa Catarina. Programa de Pós-graduação em Educação Física. Florianópolis - SC, Brasil.

3 Universidade de Pernambuco. Programa Associado de Pós-graduação em Educação Física - UPE/UFPB. Recife - PE, Brasil.

4 Universidade de Pernambuco. Programa de Pós-graduação em Hebiatria. Camaragibe $P E$, Brasil. 


\section{INTRODUÇÃO}

Os comportamentos sexuais de risco, como o não uso de preservativo e a multiplicidade de parceiros, constituem um sério problema de saúde pública. Estes comportamentos combinados com a falta de conhecimento contraceptivo e exposição a outras condutas de risco a saúde (etilismo, tabagismo, inatividade física) podem causar sérios danos à saúde, como as doenças sexualmente transmissíveis e os riscos associados à gestação precoce ou não planejada ${ }^{1-4}$.

As relações sexuais desprotegidas são mais frequentes entre os adolescentes quando comparados aos adultos ${ }^{5}$, ainda que, ao longo das últimas duas décadas, o uso de preservativos nesta população tenha crescido ${ }^{6,7}$. Apesar disso, estudos indicam que a utilização de preservativos entre os jovens não é consistente ${ }^{8-10}$. Os principais fatores predisponentes ao intercurso sexual sem uso de preservativo são: a iniciação sexual precoce, o uso de cigarros, álcool e outras drogas ${ }^{11-14}$.

A prática de atividades físicas e a participação em esportes podem favorecer a aquisição de hábitos saudáveis ${ }^{15-18}$. Entretanto, achados quanto à associação entre a prática de atividades físicas, exercícios físicos e exposição a comportamento sexual de risco são controversos ${ }^{17-19}$. Enquanto, há resultados que indicam que adolescentes com maior nível de atividade física estão menos propensos a comportamentos sexuais de risco ${ }^{15-18}$, outros estudos apontaram o inverso ${ }^{19,20}$.

Esta inconsistência de resultados encontrada nos estudos pode ser consequência de diversos fatores. A variabilidade metodológica (delineamento de pesquisa, amostragem e instrumento empregado), as diferentes abordagens nas análises estatísticas e a complexidade do fenômeno são aspectos apontados pelos autores como os possíveis responsáveis por estas divergências ${ }^{21,22}$. Deste modo, parece clara a importância de desenvolver estudos com maior representatividade populacional (regional e nacional), além de incluir no plano analítico o estudo de variáveis que possam estar mais fortemente associadas à adoção de comportamentos sexuais de risco na adolescência, inclusive com poder estatístico suficiente para controle de potenciais fatores de confusão. Diante do exposto, o objetivo do presente estudo é analisar a associação entre a prática de atividades físicas no lazer e a participação nas aulas de Educação Física com os indicadores de comportamento sexual de risco em adolescentes do estado de Pernambuco.

\section{MÉTODOS}

Este estudo é caracterizado como transversal, de base escolar e abrangência estadual. Trata-se de uma análise secundária de dados do projeto "Estilos de Vida e Comportamentos de Risco à Saúde do Estudante do Ensino Médio de Pernambuco", realizado, no período de abril a outubro de 2006. O protocolo deste projeto de pesquisa foi aprovado pelo Comitê de Ética em Pesquisa com seres humanos do Hospital Agamenon Magalhães na cidade do Recife e seguiu todas as diretrizes estabelecidas nas resoluções 196 e 251, do Conselho Nacional de Saúde.

\section{Participantes}

A população alvo foi constituída por adolescentes (14 a 19 anos), matriculados em escolas da rede pública estadual do ensino médio no Estado de Pernambuco. Considerando-se todas as dependências administrativas de âmbito federal, estadual, municipal e privada, os estudantes matriculados na rede pública estadual representavam aproximadamente $80 \%$ do total de estudantes em todo estado. O 
dimensionamento da amostra foi realizado de forma a atender os diversos objetivos do projeto, que incluíam a avaliação da exposição a dez fatores de risco à saúde, dentre os quais os indicadores de comportamento sexual de risco. Procedimentos empregados para dimensionamento amostral, seleção de participantes e poder estatístico foram apresentados em publicações prévias ${ }^{23,24}$.

\section{Instrumentos e variáveis}

O instrumento utilizado para coleta de dados foi uma versão adaptada do questionário Global School-based Student Health Survey (GSHS). Maiores informações sobre este questionário podem ser encontradas em http://www.who.int/chp/gshs/ en/. Este questionário apresenta 10 módulos de comportamentos de risco à saúde, entretanto, para o presente estudo, foram analisadas informações referentes ao comportamento sexual, atividades físicas, consumo de álcool, tabagismo e dados pessoais. $\mathrm{O}$ instrumento foi validado (validade de conteúdo e de face), submetido a estudo piloto e apresentou boa consistência nas medidas. A maioria das questões apresentou indicadores de reprodutibilidade de moderados a altos, sendo que os coeficientes de concordância variaram de 0,52 a 1,00.

Todas as informações obtidas neste estudo foram autorreferidas. As variáveis dependentes foram o "uso de preservativos" e a "quantidade de parceiros". O uso de preservativos foi obtido a partir da seguinte questão: "Na última vez que você teve relação sexual, você ou seu parceiro usou preservativo?" Foram considerados expostos a esse comportamento os estudantes que relataram não ter usado o preservativo. A quantidade de parceiros foi obtida através da questão: "Durante toda a sua vida, com quantas pessoas você já teve relação sexual?”. Foram considerados expostos os estudantes que tiveram três parceiros ou mais.

As variáveis independentes foram a prática de atividade física no lazer e a participação nas aulas de Educação Física. A prática de atividade física no lazer foi obtida através da questão: "Você realiza, regularmente, algum tipo de atividade física no seu tempo livre, como exercícios, esportes, danças ou artes marciais?”. Os estudantes foram classificados em dois grupos ( $\operatorname{sim} /$ não). A participação nas aulas de Educação Física foi definida pelo relato do número de aulas semanais que os estudantes relataram participar. Neste estudo, esta variável foi dicotomizada em dois grupos: partipantes, aqueles que relataram participar em pelo menos uma aula de Educação Física por semana, e; não participantes.

Foram considerados como potenciais fatores de confusão as seguintes variáveis: sexo (rapazes, moças); faixa etária (14-16 anos, 17-19 anos); afiliação religiosa (sim, não); escolaridade materna ( $\leq 8$ anos, 9 a 11 anos, $\geq 12$ anos); viver com os pais (sim, não); consumo de bebidas alcoólicas e tabagismo. Tais variáveis foram consideradas em estudos anteriores ${ }^{11,12,25}$.

A medida da exposição ao consumo de bebidas alcoólicas foi realizada a partir de uma única pergunta, classificando-se os estudantes que relataram ter consumido bebidas alcoólicas nos últimos 30 dias como "expostos". Similarmente, foram considerados expostos a tabagismo aqueles estudantes que referiram ter fumado nos últimos 30 dias, independentemente da frequência e intensidade da exposição.

\section{Análises estatísticas}

A tabulação dos dados foi realizada no programa EpiData versão 3.1 e as análises estatísticas foram realizadas mediante utilização do pacote estatístico STATA (versão 10). As análises descritivas foram efetuadas pela distribuição de frequências e as análises bivariadas pela aplicação do teste de Qui-quadrado. As análises 
de associação entre as variáveis independentes e dependentes foram conduzidas mediante utilização de regressão logística binária separadamente para cada um dos desfechos (não usar preservativo e ter tido três ou mais parceiros). Inicialmente, realizaram-se análises brutas e, depois, as multivariáveis que foram conduzidas a fim de controlar potenciais fatores de confusão.

\section{RESULTADOS}

Do total de adolescentes matriculados e presentes nas turmas sorteadas nas 76 escolas investigadas (4.269), 55 sujeitos se recusaram a participar e sete foram excluídos devido a dados incompletos/inconscientes no questionário. A amostra final ficou constituída por 4.207 adolescentes (59,8\% do sexo feminino). Dentre as variáveis analisadas neste estudo, as taxas de questões sem resposta não ultrapassou 1,6\%. A média de idade dos sujeitos foi 16,8 anos $(\mathrm{DP}=1,4)$. Verificou-se que $85,5 \%$ dos adolescentes tinham alguma afiliação religiosa e 63,6\% relataram morar com os pais. Também foi observado que a maioria dos estudantes foi considerada não exposta ao consumo de álcool $(69,6 \%)$ e ao tabagismo $(92,4 \%)$. As características sociodemográficas e comportamentais da amostra por sexo estão apresentadas na Tabela 1 .

Tabela 1 - Características demográficas, socioeconômicas e fatores relacionados à saúde dos adolescentes ( $n=4.207)$ do estado de Pernambuco, Brasil, 2006.

\begin{tabular}{|c|c|c|c|c|}
\hline \multirow{2}{*}{ Variável } & \multicolumn{2}{|c|}{ Rapazes } & \multicolumn{2}{|c|}{ Moças } \\
\hline & $\%$ & $\mathrm{n}$ & $\%$ & $n$ \\
\hline \multicolumn{5}{|l|}{ Faixa etária (anos)* } \\
\hline $14-16$ & 35,4 & 598 & 46,4 & 1.165 \\
\hline $17-19$ & 64,6 & 1.089 & 53,6 & 1.346 \\
\hline \multicolumn{5}{|c|}{ Escolaridade materna $\$$} \\
\hline$\leq 8$ anos & 69,4 & 1.086 & 74,5 & 1.771 \\
\hline $9-11$ anos & 22,5 & 352 & 20,2 & 480 \\
\hline$\geq 12$ anos & 8,1 & 127 & 5,3 & 126 \\
\hline \multicolumn{5}{|l|}{ Afiliação religiosa" } \\
\hline Não & 19,4 & 325 & 11,2 & 281 \\
\hline Sim & 80,6 & 1.354 & 88,8 & 2.221 \\
\hline \multicolumn{5}{|l|}{ Morar com os pais ${ }^{\S}$} \\
\hline Sim & 68,1 & 1.130 & 60,6 & 1.510 \\
\hline Não & 31,9 & 529 & 39,4 & 981 \\
\hline \multicolumn{5}{|l|}{ Consumo de álcool ${ }^{\ddagger}$} \\
\hline Não exposto & 61,4 & 1.030 & 75,2 & 1.885 \\
\hline Exposto & 38,6 & 648 & 24,8 & 622 \\
\hline \multicolumn{5}{|l|}{ Tabagismo } \\
\hline Não exposto & 90,2 & 1.514 & 93,8 & 2.345 \\
\hline Exposto & 9,8 & 165 & 6,2 & 155 \\
\hline
\end{tabular}

Dados sem resposta: ${ }^{*} n=9,{ }^{\$} n=265,{ }^{9} n=26,{ }^{\S} n=57,{ }^{\ddagger} n=22,{ }^{*} n=28$.

Em relação à prevalência dos indicadores de comportamento sexual de risco, observou-se que 12,4\% (IC95\%: 11,4-13,4) dos adolescentes relataram não ter usado preservativo durante a última relação sexual. Verificou-se ainda que 20,9\% (IC95\%: 19,7-22,1) dos estudantes referiram ter três ou mais parceiros.

Por outro lado, a prática de atividade física no lazer e a participação nas aulas de Educação Física foram observadas, respectivamente, em 61,8\% (IC95\%: 60,3-63,2) e 35,1\% (IC95\%: 33,7-36,6) dos estudantes. Os resultados da associação entre a 
prática de atividade física no lazer e a participação nas aulas de Educação Física com os indicadores de comportamento sexual de risco estão apresentados na Figura 1.

Não usar preservativo

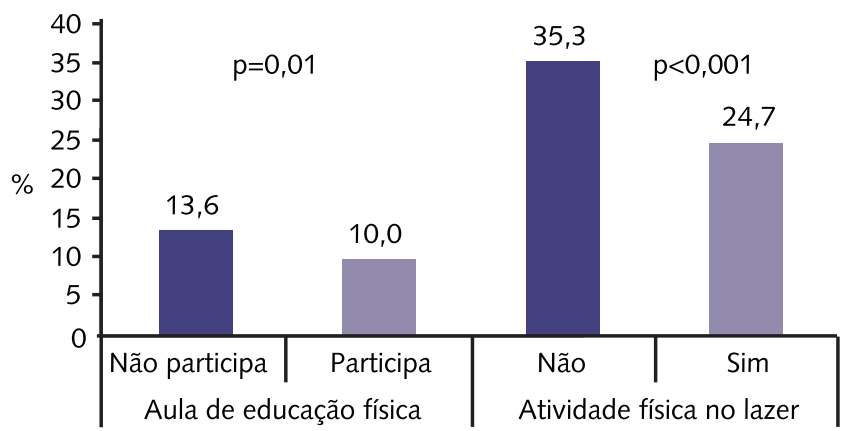

(A)

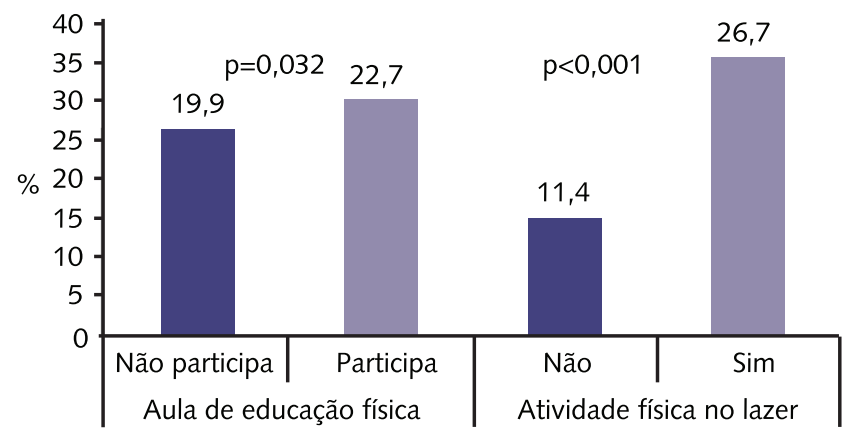

Figura 1 - Prevalência de adolescentes expostos ao não uso de preservativo (A) e, intercurso sexual com três ou mais parceiros de acordo com a participação nas aulas de Educação Física e atividade física no lazer (B).

Após ajustamento, observou-se que a participação nas aulas de Educação Física foi um fator inversamente associado ao intercurso sexual sem uso de preservativo. Opostamente, a prática de atividade física no lazer apresentou associação direta com maior número de parceiros sexuais (Tabela 2).

Tabela 2 - Análise bruta e ajustada para associação entre o não uso de preservativo e ter três ou mais parceiros com a participação nas aulas de Educação Física em adolescentes.

\begin{tabular}{|c|c|c|c|c|c|}
\hline Variáveis independentes & Categorias & OR bruto & $\mathrm{p}$ & OR ajustado* & $\mathrm{p}$ \\
\hline \multicolumn{6}{|c|}{ Desfecho: Não usar preservativo } \\
\hline \multirow{2}{*}{$\begin{array}{l}\text { Participação nas aulas } \\
\text { de EF }\end{array}$} & Não participa & 1 & \multirow[b]{2}{*}{0,001} & 1 & \multirow[b]{2}{*}{0,007} \\
\hline & Participa & $0,71(0,58-0,87)$ & & $0,75(0,60-0,92)$ & \\
\hline \multirow[t]{2}{*}{ Atividade física no lazer } & Não & 1 & & 1 & \\
\hline & Sim & $1,02(0,84-1,23)$ & 0,833 & $0,85(0,69-1,04)$ & 0,119 \\
\hline & \multicolumn{5}{|c|}{ Desfecho: Ter três ou mais parceiros } \\
\hline \multirow{2}{*}{$\begin{array}{l}\text { Participação nas aulas } \\
\text { de EF }\end{array}$} & Não participa & 1 & \multirow[b]{2}{*}{0,032} & 1 & \multirow[b]{2}{*}{0,347} \\
\hline & Participa & $1,18(1,01-1,38)$ & & $1,09(0,91-1,32)$ & \\
\hline \multirow[t]{2}{*}{ Atividade física no lazer } & Não & 1 & & 1 & \\
\hline & Sim & $2,83(2,37-3,38)$ & $<0,001$ & $1,67(1,35-2,06)$ & $<0,001$ \\
\hline
\end{tabular}

*Ajustado para sexo, faixa etária, afiliação religiosa, morar com os pais, consumo de álcool e tabagismo.

\section{DISCUSSÃO}

Os principais achados deste estudo foram: a identificação de associação inversa entre a participação nas aulas de Educação Física e o intercurso sexual sem uso de preservativo; e a associação direta entre a prática de atividade física no lazer e o intercurso sexual com três ou mais parceiros. Este estudo também revelou que o intercurso sexual com três ou mais parceiros ao longo de vida foi um indicador de comportamento sexual de risco mais prevalente do que não usar preservativos.

Destaca-se dentre os principais pontos positivos do estudo, o tamanho e a estratégia de seleção da amostra que asseguraram razoável precisão às análises e representatividade estadual da população de adolescentes estudantes do ensino médio. As informações foram obtidas mediante utilização de um questionário previamente testado e com boa consistência nas medidas. Os aplicadores foram previamente 
treinados para padronizar as medidas e reduzir os erros intra e interavaliador. Também é importante salientar que não foi localizado nas bases de dados do Pubmed, Scopus e Scielo referência a qualquer estudo de base escolar envolvendo adolescentes brasileiros e que possa ser considerado congênere ao relatado no presente artigo.

Apesar dos pontos fortes mencionados, destaca-se que o estudo também apresenta algumas limitações. Mesmo com garantia de anonimato e sigilo das informações fornecidas, ainda é possível que alguns adolescentes tenham omitido o "intercurso sexual sem uso de preservativo" e a "quantidade de parceiros sexuais no curso da vida”, o que pode ser considerado um viés de informação, conduzindo a uma estimativa errônea da prevalência de exposição a estas condutas de risco. Além disso, o delineamento adotado permite a ocorrência de viés de causalidade reversa. Por isso, a interpretação das associações que foram verificadas deverá ser efetuada com cautela. Outro ponto a ser considerado é que os adolescentes matriculados em escolas privadas e aqueles que não estavam frequentando a escola não foram incluídos no estudo, limitando a extrapolação dos resultados que não pode ser generalizada para toda a população adolescente.

A análise da associação entre intercurso sexual e prática de atividade física em adolescentes está documentada em alguns estudos internacionais. Levantamentos realizados com jovens americanos revelaram que a participação em esportes está associada à maior chance de exposição à conduta sexual de risco (parceiros múltiplos) $)^{19,20}$. Os resultados de tais levantamentos são convergentes em relação ao observado no presente estudo, onde a prevalência de intercurso com três ou mais parceiros durante a vida foi maior entre os adolescentes fisicamente ativos no lazer. Uma das possíveis explicações para este achado pode residir no tipo de atividade física que é usualmente praticado pelos adolescentes que envolvem esportes e práticas corporais nas quais a exposição a risco (velocidade, altura, queda, dentre outros) constitui um ingrediente essencial à aderência dos jovens. Outra possível explicação é o maior contato físico e interação que ocorre entre jovens fisicamente ativos e que podem conduzir a experiência sexual precoce. Todavia, estas hipóteses estão baseadas em especulações teóricas não havendo, neste momento, dados empíricos que permitam testá-las, razão pela qual mais estudos são necessários.

Não identificamos na literatura especializada estudos prévios que tenham explorado a inter-relação entre a participação nas aulas de Educação Física e a exposição a comportamentos de risco à saúde. No presente estudo, observou-se que a participação nas aulas de Educação Física está associada a menor chance de intercurso sexual sem uso de preservativo. Este resultado é encorajador, pois apesar das criticas e questionamentos ao papel que a Educação Física escolarizada pode assumir na abordagem de temas e questões relacionadas à saúde, os resultados sugerem que este componente curricular pode ter um importante papel na promoção de condutas relacionadas à saúde na adolescência.

A Educação Física é considerada uma área privilegiada para abordagem de temas relacionados à saúde, seja por seus conteúdos que prezam por uma postura autônoma na escolha de atividades para manutenção e aquisição da saúde ou porque as aulas de Educação Física são espaços onde os professores podem estimular o desenvolvimento de conhecimentos, atitudes e habilidades relacionados ao cuidado com o corpo e a saúde ${ }^{26}$.

A elevada prevalência de adolescentes expostos a comportamentos de risco à saúde indica também a necessidade de reinserção da discussão sobre sexualidade no currículo escolar e nas ações de atenção à saúde do escolar. Isto é particularmente importante ao considerar o crescimento dos casos de gravidez indesejada e 
contaminação pelo vírus da imunodeficiência humana (HIV) em adolescentes ${ }^{27}$.

Os achados anteriormente discutidos devem ser generalizados e interpretados com cautela, particularmente no tocante a um presumido risco da prática de atividades físicas em relação à adoção de comportamentos sexuais de risco. Este cuidado se justifica pela impossibilidade de estabelecimento de causalidade diante do delineamento de investigação adotado e porque outros estudos apontaram resultados divergentes em relação aos achados neste estudo. Acredita-se que em determinados subgrupos populacionais, a prática de atividades físicas pode não ter associação com comportamentos sexuais de riscos ou tal associação pode ser mediada por fatores que ainda não foram suficientemente investigados ou esclarecidos ${ }^{16,19}$.

Diante do exposto, sugere-se o desenvolvimento de mais estudos, inclusive com adoção de delineamentos longitudinais e em outras regiões do país, a fim de verificar se os achados aqui relatados se confirmam ou se podem ser explicados por fatores não controlados na presente investigação. Além disso, seria importante o desenvolvimento de abordagens qualitativas de pesquisa (exemplo: estudo de grupos focais) que permitam um maior esclarecimento sobre os mecanismos que podem explicar as associações relatadas neste estudo.

Diante dos resultados apresentados, pode-se concluir que tanto a participação nas aulas de Educação Física quanto a prática de atividades físicas no lazer são fatores associados a indicadores de comportamento sexual de risco em adolescentes pernambucanos. Todavia, a participação nas aulas de Educação Física foi associada a menor chance de intercurso sexual sem uso de preservativo, enquanto a prática de atividades físicas no lazer foi associada a maior chance de intercurso sexual com três ou mais parceiros. Estes resultados permaneceram inalterados mesmo após ajustamento das análises para potenciais variáveis de confusão (sexo, faixa etária, afiliação religiosa, morar com os pais, consumo de álcool e tabagismo). Os achados desta investigação adicionam ao corpo de conhecimento disponível, evidências de que a participação nas aulas de Educação Física e a prática de atividades físicas podem representar contextos favoráveis ao desenvolvimento de intervenções para promoção de comportamentos relacionados à saúde em adolescentes.

\section{Financiamento}

Projeto de pesquisa que derivou a realização do presente estudo recebeu suporte da Coordenação de Aperfeiçoamento de Pessoal de Nível Superior (CAPES), Fundação de Amparo à Ciência e Tecnologia do Estado de Pernambuco (FACEPE) e Conselho Nacional de Desenvolvimento Científico e Tecnológico (CNPq).

\section{REFERÊNCIAS}

1. Barbosa EMS, Frota MLM, Silva K, Petribu KCL, Amorim VCSA. Comportamento Sexual e reprodutivo de adolescentes brasileiros: análise das variáveis adotadas nas pesquisas científicas. Neurobiologia 2008;71:09-19.

2. Leite IC, Rodrigues RN, Fonseca MC. Fatores associados com o comportamento sexual e reprodutivo entre adolescentes das regiões Sudeste e Nordeste do Brasil. Cad Saude Publica 2004;20(2):474-81.

3. Raffaelli M, Crockett LJ. Sexual risk taking in adolescence: the role of self-regulation and attraction to risk. Dev Psychol 2003;39(6):1036-46.

4. Sterling SP, Sadler LS. Contraceptive use among adolescent Latinas living in the United States: the impact of culture and acculturation. J Pediatr Health Care 2009;23(1):19-28.

5. Camargo BV, Botelho LJ. Aids, sexualidade e atitudes de adolescentes sobre prevenção contra o HIV. Rev Saude Publica 2007;41(1):61-68. 
6. Almeida MCC, Aquino EML, Gaffikinb L, Magnanic RJ. Contraceptive use among adolescents at public schools in Brazil. Rev Saude Publica 2003;37(5):566-75.

7. Paiva V, Calazans G, Venturi G, Dias R. Idade e uso de preservativo na iniciação sexual de adolescentes brasileiros. Rev Saude Publica 2008;42(suppl 1):45-53.

8. Fergus S,Zimmerman M, Caldwell C. Growth trajectories of sexual risk behavior in adolescence and young adulthood. Am J Public Health 2007;97(6):1096-101.

9. Hirsl-Hećej V, Stulhofer A. Urban adolescents and sexual risk taking. Coll Antropol 2001;25(1):195-212.

10. Narring F, Wydler H, Michaud PA. First sexual intercourse and contraception: a crosssectional survey on the sexuality of 16-20-year-olds in Switzerland. Schweiz Med Wochenschr 2000;130(40):1389-98.

11. East PL, Khoo ST. Longitudinal pathways linking family factors and sibling relationship qualities to adolescent substance use and sexual risk behaviors. J Fam Psychol 2005;19(4):571-80.

12. Moser AM, Reggiani C, Urbanetz A. Comportamento sexual de risco entre estudantes universitárias dos cursos de ciências da saúde. Rev Assoc Med Bras 2007;53(2):116-21.

13. Robinson KL, Telljohann SK, Price JH. Predictors of sixth graders engaging in sexual intercourse. J Sch Health 1999;69(9):369-75.

14. Santelli JS, Brener ND, Lowry R, Bhatt A, Zabin LS. Multiple sexual partners among U.S. adolescents and young adults. Fam Plann Perspect 1998;30(6):271-75.

15. Eitle TM, Eitle DJ. Just don't do it: High school sports participation and young female adult sexual behavior. Sociol Sport J 2002;19(4):403-18.

16. Kulig K, Brener ND, Mcmanus T. Sexual activity and substance use among adolescents by category of physical activity plus team sports participation. Arch. pediatr. adolesc. med 2003;157(9):905-12.

17. Sabo DF, Miller KE, Farrell MP, Melnick MJ, Barnes GM. High school athletic participation, sexual behavior and adolescent pregnancy: a regional study. J Adolesc Health 1999;25(3):207-16.

18. Savage MP, Holcomb DR. Adolescent female athletes' sexual risk-taking behaviors. J Youth Adolesc 1999;28(5):595-60.

19. Faurie C, Pontier D, Raymond M. Student athletes claim to have more sexual partners than other students. Evol Hum Behav 2004;25(1):1-8.

20. Wetherill RR, Fromme K. Alcohol use, sexual activity, and perceived risk in high school athletes and non-athletes. J Adolesc Health 2007;41(3):294-301.

21. Bungay H, Vella-Burrows T. The effects of participating in creative activities on the health and well-being of children and young people: a rapid review of the literature. Perspect Public Health 2013;133:44-52.

22. Johnson KE, Taliaferro LA. Health behaviors and mental health of students attending alternative high schools: A review of the research literature.J Spec Pediatr Nurs 2012;17:79-97.

23. Santos CM et al. Prevalence of physical inactivity and associated factors among adolescents commuting to school. Cad Saude Publica 2010;26(7):1419-30.

24. Tassitano RM et al. Enrollment in physical education is associated with health-related behavior among high school students. J Sch Health 2010;80(3):126-133.

25. Dittus PJ, Jaccard J. Adolescents' perceptions of maternal disapproval of sex: relationship to sexual outcomes. J Adolesc Health 2000;26(4):268-78.

26. Brasil. Ministério da Educação. Secretaria de Educação Fundamental. Parâmetros Curriculares Nacionais/Educação Física: terceiro e quarto ciclos do ensino fundamental. Brasília: MEC/ SEF;1998.

27. Altmann H. Orientação sexual nos parâmetros curriculares nacionais. Estud. fem 2001;9(2):575-85.

Endereço para Correspondência Rua Arnóbio Marques, 310, Campus Universitário HUOC/ESEF

Santo Amaro, Recife - PE, 50100-130 E-mail: maurovgb@gmail.com Telefone: +55 81 3183-3376

Recebido 13/07/2013 Revisado 09/10/2013 Aprovado 17/10/2013 